Table 1 The four trials analyzed by Chung et al. ${ }^{1}$

\begin{tabular}{|c|c|c|c|c|c|c|}
\hline Trial & Immunogen & Vector & $\begin{array}{l}\text { Immunogen } \\
\text { administration }\end{array}$ & Participants & Start date & Outcome \\
\hline \multirow[t]{2}{*}{ RV144 } & Prime:gag-pr-gp41-gp120 & Canarypox & $0,4,12,24$ & \multirow[t]{2}{*}{16,403} & \multirow[t]{2}{*}{ October 2003} & \multirow{2}{*}{$\begin{array}{l}\text { Some }(31.2 \%) \\
\text { efficacy }\end{array}$} \\
\hline & Boost: gp120 (clade B,E) & Subunit proteins & 12,24 weeks & & & \\
\hline VAX003 & gp120 (clade B, E) & Subunit proteins & $0,1,6,12,18,24,30$ months & 2,500 & March 1999 & No efficacy \\
\hline \multirow[t]{2}{*}{ HVTN204 } & Prime: Env (clades $A, B, C$ ), gagB, polB, nefB & DNA & $0,1,2$ months & 480 & September 2005 & No efficacy \\
\hline & Boost:: Env (clades $A, B, C$ ), gagB, polB, & rAd5 & 6 months & & & \\
\hline IPCAVD 001 & Env clade $A$ & rAd26 & 0 weeks & 60 & February 2008 & No efficacy \\
\hline
\end{tabular}

Serum samples (30 vaccinees for each trial) were taken 2 weeks after the last immunization.

of Fc-related vaccine signatures, there are practical and methodological concerns that will have to be taken into consideration when undertaking such analyses. For example, the assays used for the Fc effector functions are not easy to standardize across laboratories. Future studies would benefit from the use of blinded samples. It would also be of interest to test samples from lymphoid tissues, the major site of HIV-1 replication, in addition to blood samples. The results of systems analyses depend on the breadth and depth of the training data set and often cannot be generalized. Thus, the authors' conclusions may not hold for other immunogens or viruses. Finally, in the RV144 trial, only very limited protection was achieved and less than $1 \%$ of all participants were infected, so the significance of any identified correlate of protection remains to be determined.

Nevertheless, a systems serology approach could be readily applied to other trials and expanded to include additional parameters, such as sequence analysis of antibodyomes ${ }^{4}$ and molecular signatures of vaccines. Such studies might also be designed to address the important question of how to predict the efficacy of a novel vaccine based on in vitro studies or animal models. Finding the immunological correlates of vaccine protection for HIV-1 infection by considering qualitative and quantitative immune responses could help guide us to the next generation of vaccines ${ }^{11}$.

\section{COMPETING FINANCIAL INTERESTS}

The authors declare competing financial interests: details are available in the online version of the paper.

1. Chung, A.W. et al. Cell 163, 988-998 (2015).

2. Shibata, R. et al. Nat. Med. 5, 204-210 (1999).

3. Caskey, M. et al. Nature 522, 487-491 (2015).

4. Xiao, X. et al. Biochem. Biophys. Res. Commun. 390 404-409 (2009).

5. Dimitrov, D.S. MAbs 2, 347-356 (2010).

6. Haynes, B.F., Kelsoe, G., Harrison, S.C. \& Kepler, T.B. Nat. Biotechnol. 30, 423-433 (2012).

7. Rerks-Ngarm, S. et al.; MOPH-TAVEG Investigators. N. Engl. J. Med. 361, 2209-2220 (2009).

8. Haynes, B.F. et al. N. Engl. J. Med. 366, 1275-1286 (2012).

9. Yates, N.L. et al. Sci. Transl. Med. 6, 228ra39 (2014).

10. Chung, A.W.E. et al. Sci. Transl. Med. 6, 228ra38 (2014).

11. Corey, L. et al. Sci. Transl. Med. 7, $310 r v 7$ (2015).

\title{
Getting rid of PERVs
}

CRISPR-Cas technology has been used in a broad range of applications, including multiplexing. Up to five genes have been targeted in the same cell ${ }^{1}$, but whether this number could be extended by much was not clear. In a new demonstration of the capabilities of the CRISPR-Cas system, George Church, Luhan Yang and colleagues recently reported simultaneously editing 62 related genes in pig cells ${ }^{2}$.

Engineering the genome of pigs is not a new idea. A strong incentive for altering the genetic makeup of these animals is to make them more suitable as donors for xenotransplantation. Pig organs are roughly the same size as those of humans and share similar vascular architecture. Tens of thousands of individuals could receive life-saving transplants every year if the remaining hurdles in xenotransplantation were overcome. One such challenge is the potential transmission of porcine endogenous retroviruses (PERVs) to humans.

Aiming to inactivate PERVs, Church, Yang and colleagues began by determining how many PERV elements are present in the genome of pig cells, and identified 62. Then, they designed two Cas9 guide RNAs that would target the pol gene, an essential and conserved PERV gene, in all 62 PERVs. They expressed the guide RNAs and Cas9 in PK15 cells, a pig kidney epithelial cell line, and sequenced clones to determine the extent of editing that had occurred. They found a small number of clones that had up to $100 \%$ disruption of the pol gene, along with evidence suggesting that this high efficiency was due to a mechanism in which previously edited pol genes were used as templates to disrupt wild-type pol genes. Elucidating the intricacies of this mechanism may uncover interesting biology and further potential applications of the approach.

"This illustrates the power of the CRISPR technology," says Randall Prather, of the University of Missouri. "A few years ago we wouldn't have thought of doing this because inactivating 62 genes, or even a family of 10 genes, would have been quite complicated with traditional homologous recombination."

Editing 62 genes, even if closely related, is certainly a feat, and the engineered cells showed a 1,000-fold reduction in PERV transmission to human cells. That said, whether PERV transmission is likely to be a problem in xenotransplantation is not clear. Kevin Wells, also from the University of Missouri, says that PERV transmission was on the list of things to examine, but probably not at the top. "We don't know what the actual risk is," he says. Although there is evidence that PERV transmission can occur in vitro, "no one has observed transmission of PERVs in experiments where a nonhuman primate received a pig organ," says Wells. In addition, alternative approaches could be used, such as employing pig lines that do not produce human-tropic, replication-competent PERVs ${ }^{3}$.

Organ rejection continues to be the major hurdle in xenotransplantation. Although acute organ rejection has been addressed, immune rejection is still a considerable problem. On that front, Wells points out that CRISPR-Cas9 genome editing could be used to

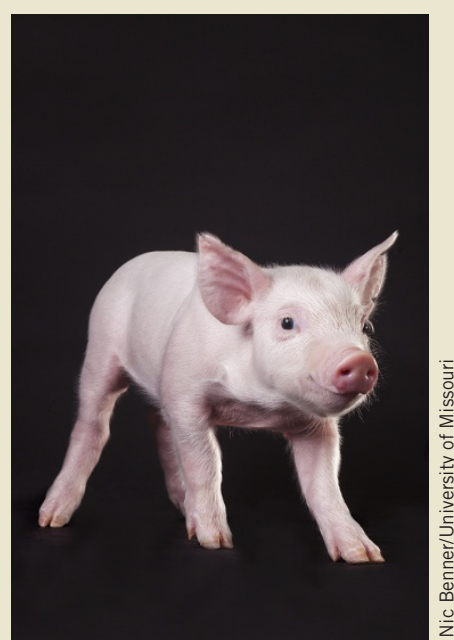

The 62 endogenous porcine retrovirus genes have been inactivated in pig cells. Are piglets next?

target pig genes encoding antigenic carbohydrates or proteins. Inactivation of porcine circulating proteins that don't interact well with their human counterparts could also help efforts to make xenotransplantation a reality.

\section{Irene Jarchum, Associate Editor}

1. Wang, H. et al. Cell 153 910-918 (2013).

2. Yang, L. et al. Science 350, 1101-1104 (2015).

3. Oldmixon, B.A. et al. J. Virol. 76, 3045-3048 (2002). 